\title{
Authors Contributing to Volume B45
}

All authors contributing to Volume B45 are listed alphabetically with the part and page numbers. A full author index will also be published.

Abrahams, I., (5) 457

Abrahams, S.C., (1) $26,34,52$ (3) 228, (4) 364

Albano, V.G., (1) 60

Albertsson, J., (1) 34

Almeida, M.J.M. de, (6) 549

Andersen, L., (4) 344

Anderson, A.A., (2) 124

Anthoni, U., (6) 562

Ariel, S., (2) 153

Baert, F., (3) 276

Bailey, P.D., (5) 482

Barbier, J., (3) 201

Barlow, R.B., (4) 396

Bartels, K.S., (2) 190

Bates, P.A., (2) 147

Bekkum, H. van, (4) 423

Belgrand, T., (4) 404

Berndt, J., (1) 69

Bestmann, H.J., (3) 303

Bhandary, K.K., (3) 306

Bigi, A., (3) 247

Blaton, N.M.. (1) 107

Blessing, R.H., (2) 200

Bochu, C., (3) 276

Boeyens, J.C.A., (6) 577, 581

Böhlen, K. von, (2) 190

Boswell, F.W., (6) 529

Braga, D., (1) 60 , (4) 378

Bruce, P.G., (5) 457

Brückner, S., (3) 247

Budkowski, A., (6) 529

Bürgi, H.B. (4) 383

Burzlaff, H., (3) 303

Cameron, T.S., (2) 167

Carré, M.C., (2) 178

Caubère, P., (2) 178

Chantler, C.T., (3) 290

Chapuis, G., (1) 20

Cheary, R.W.., (3) 205

Christophersen, C., (6) 562

Ciunik, Z., (5) 512

Clark, R.J.H., (2) 147

Cody, V., (2) 172

Coppens, P., (3) 298

Costa, M.M.R., (6) 549

Couture, A., (3) 276

Craven, B.M., (3) 270

Croud, V.B., (2) 147

Curtin, D.Y., (4) 411

Danz, H., (2) 190

David, W.I.F., (5) 457

Dawes, H.M., (2) 147

Depmeier, W., (6) 555

De Ranter, C.J., (1) 107

Desiraju, G.R., (5) 473

Dijkstra, B.W. (5) 488

Dodson, E.J., (5) 482

Dodson, G., (1) 99

Doi, M.. (5) 505

Dorset, D.L., (3) 283

Dravid, V.P., (3) 218

Dunitz, J.D., (3) 312

Ebey, W.J., (1) 93

Etxebarria, I., (6) 566

Evans, D.G., (6) 577, 581

Evans, S.V., (2) 159, (5) 500

Expeleta, J.M., (6) 566

Feil, D., (4) 359

Figgis, B.N., (3) 232, 240

Fleet, M.E., (3) 201
Foulon, M., (4) 404

Frolow, F., (2) 190

Fujinaga, M., (5) 488

Gajhede, M., (6) 562

Gammon, R.E., (5) 482

Gao, Y., (3) 298

Garcia-Garibay, M., (2) 153

Gáspár, Zs., (4) 452

Gavezzotti, A., (5) 473

Gazzano, M., (3) 247

Geoffroy, P., (2) 178

Go, K., (3) 306

Godefroy, G., (4) 370

Gomes, E., (4) 348

Gomez de Anderez, D.,

(5) 482, (6) 591

Görbiz, C.H., (4) 390

Gors, C., (4) 404

Graser, F., (1) 69

Grebille, D., (4) 370

Grepioni, F., (1) 60, (4) 378

Gros, P., (5) 488

Habash, J., (5) 482, (6) 591

Hädicke, E., (1) 69

Halfon, Y. (2) 190

Hasebe, K., (2) 199

Ilashimoto, H., (2) 129

Haufe, G., (4) 416

Hedoux, A., (4) 370

Helliwell, J.R., (5) 482 ,

(6) 591

Helliwell, M., (5) 482 ,

(6) 591

Hermansson, K., (3) 252

Higgins N.A., (4) 437,442

Hol, W.G.J., (5) 488

Hope, H., (2) 190

Howard, J.A.K., (4) 396

Hu, H., (3) 283

Hümmer, K., (3) 303

Hursthouse, M.B., (2) 147

Hyde, B.G., (2) 136

Ianelli, S., (2) 178

Ihringer, J., (1) 26

In, Y.. (5) 505

Inoue, M., (5) 505

Ishida, T., (5) 505

Jamart-Grégoire, B., (2) 178

Jansen, J.C., (4) 423

Jaskólski, M., (1) 78

Jaud, J., (4) 370

Jeffrey, G.A., (4) 447

Ji, X., (1) 93

Jochem, K., (2) 167

Johnson, O., (4) 396

Jorgensen, J.D., (3) 212

Kalk, K.H., (5) 488

Kamer, G., (1) 85

Keszler, D.A., (1) 13

Kitano, Y., (i) 6

Klebe, G." (1) 69

Koellner, K., (4) 396

Komura, Y., (1) 6

Koningsveld, H. van,

(4) 423

Konno, M. (2) 142

Koshiji, N., (5) 467

Kratky, C., (2) 190

Krebs, B., (3) 257

Kucharczyk, D., (6) 529
Kucharski, E.S., (3) 232, 240

Kvick, А̊., (1) 34

Lalancette, R.A., (6) 596

Lamiot, J., (3) 276

Langer, V., (4) 344

Lawson, A.C., (3) 212

Lefebvre, J., (3) 323

Leligny, H., (2) 113, 117

Li, F.H., (2) 129

Liao, J.H. (1) 65

Linden, A., (2) 167

Luft, J., (2) 172

Luger, P., (5) 512

Luo, M. (1) 85

Luty, T., (3) 323

Lyman, C.E., (3) 218

Machida, K., (2) 162

Madariaga, G., (5) 462 ,

(6) 566

Makowski I., (2) 190

Maluszynska, H., (4) 447

Mardix, S., (4) 454

Marinković, V., (6) 529

Marsh, P., (1) 26, 52, (4) 364

Mashiyama, H., (2) 199

(5) 467

Maslen, E.N., (1) 45, (3) 290

Mayer, J., (4) 355

McMullan, R.K., (3) 270

Mierzejewski, A., (3) 323

Mo, $[\because$, (3) 26$]$

Monier, J.C. (2) 113,117

More, M., (4) 404

Morosin, B., (3) 212

Morris, D.G., (2) 167

Mouchel, B., (3) 276

Muller, M., (3) 323

Myszka, H., (5) 512

Nardelli, M., (2) 178

Nardin, G., (6) 521

Nielsen, P.H., (6) 562

Notis, M.R., (3) 218

Odou, G., (3) 323

Ohba, S., (4) 432

Okamoto, T., (2) 142

Olovsson, I., (1) 78

Paciorek, W.A., (6) 566

Padmanabhan, K., (4) 411

Paixão, J.A., (6) 549

Paul, I.C., (4) 411

Paulsen, H., (5) 512

Peeters, O.M., (1) 107

Pérez-Mato, J.M., (5) 462

(6) 566

Prodan, A., (6) 529

Randaccio, L., (6) 521

Ravez, J., (4) 364

Reynolds, P.A., (3) 232, 240

Richardson, M.F., (3) 312

Ripamonti, A., (3) 247

Roof, R.B., (3) 212

Rossmann, M.G., (1) 85

Roth, D., (3) 303

Rothammel, W., (3) 303

Roveri, N., (3) 247

Russell, M.A., (4) 416

Saito, Y., (4) 432

Savage, H., (1) 99

Schäfer, G., (3) 303
Scheffer, J.R., (2) 153

Schirber, J.E., (3) 212

Schwetlik, G., (3) 257

Scrimgeour, S.N., (3) 261

Shcpelev, Y.F., (2) 124

Shepherd, A.G., (2) 167

Shirotani, I., (2) 142

Shoemaker, C.B., (1) 13

Shoemaker, D.P., (1) 13

Sim, G.A., (4) 416

Slifer, P.B. (6) 596

Smiatacz, Z, (5) 512

Smolin, Y.I., (2) 124

Spadaccini, N., (1) 45

Spcziali, N.L., (1) 20

Squadrito, R., (3) 205

Steurer, W., (4) 355 , (6) 534 ,

Strömberg, A., (4) 344

Strömberg, D., (4) 344

Sung. C.M., (3) 218

Taga, T., (2) 162

Takata, M., (1) 6

Takazawa, H., (4) 432

Taniguchi, Y. (1) 40

Tanisaki, S., (2) 199

Tellgren, R., (3) 252

Thomas, N.W., (4) 337

Thomas, P.A., (4) 348

Thomas, S.A., (3) 247

Thompson, H.W., (6) 596

Thompson, J.G. (2) 136

Trotter, J., (2) 153, 159 ,

(5) 500

Tuinstra, F., (4) 423

Uszyński, 1., (6) 529

van der Helm, D., (1) 93

Velders, G.J.M. (4) 359

Verlinde, C.L., (1) 107

Vemon, J., (6) 591

Von Drecle, R.B., (6) 542

Vriend, G., (1) 85

Walton, D.C., (4) 396

Wang. Y., (1) 65

Webster, P., (2) 190

Wechsler, B.A., (6) 542

Wecker, E., (3) 303

West, A.R. (5) 457

Wienkenhöver, M., (3) 257

Williams, R.V., (1) 93

Wills, R.J., (2) 147

Winther, S., (3) 261

Withers, R.L., (2) 136

Wittmann, H.G., (2) 190

Wlodawer, A., (1) 99

Wu, X.J., (2) 129

Yamada, T., (1) 40

Yamamoto, N., (2) 162

Yanagisawa, I., (5) 505

Yang. Q.-C. (3) 312

Ye, H.Q., (i) 1

Yonath, A., (2) 190

Yoshida, K., (1) 40

Zangrando, E., (6) 521

Zhu, J., (1) 1

Zuñiga, F.J., (5) 462 ,

(6) 566 